\title{
Linx
}

Revue des linguistes de l'université Paris X Nanterre

$10 \mid 1998$

L'indicible et ses marques dans l'énonciation

\section{La ponctuation et les reprises de l'indicible}

\section{Véronique Dahlet}

\section{OpenEdition}

\section{Journals}

Édition électronique

URL : http://journals.openedition.org/linx/952

DOI : $10.4000 /$ linx.952

ISSN : 2118-9692

\section{Éditeur}

Presses universitaires de Paris Nanterre

\section{Édition imprimée}

Date de publication : 1 juillet 1998

Pagination : 21-29

ISSN : 0246-8743

\section{Référence électronique}

Véronique Dahlet, «La ponctuation et les reprises de l'indicible », Linx [En ligne], 10 | 1998, mis en ligne le 05 juillet 2012, consulté le 02 mai 2019. URL : http://journals.openedition.org/linx/952 ; DOI :

10.4000/linx.952

Ce document a été généré automatiquement le 2 mai 2019.

Département de Sciences du langage, Université Paris Ouest 


\title{
La ponctuation et les reprises de l'indicible
}

\author{
Véronique Dahlet
}

la virgule viendra où je me noierai pour de bon

Beckett

L'indicible, en principe, serait ce qui par définition échappe au langage, ce sur quoi le langage n'aurait pas de prise. Comment la linguistique du discours pourrait donc s'en saisir? Mais en même temps, quoi d'autre que le langage, à travers lui et par lui, pourrait en accuser les manifestations? Si l'indicible se niche dans le dire, on admettra qu'il se manifeste par des formes, qui sont moins celles du silence (telles que le blanc ou la suspension) que celles de substitution. Les formes de l'indicible sont des formes souvent pleines, qui sont mises-là-pour, en lieu et place de ce qui ne peut se dire : organiquement, l'indicible procède en toute rigueur de la même manière que la métaphore.

2 La ponctuation constitue-t-elle une forme de l'indicible? Ensemble de signes idéographiques qui entrent en interaction avec les signes linguistiques pour produire le sens, la ponctuation semble se soumettre à une constante : ou bien elle opère de façon coorientante, ou bien de façon dés-orientante par rapport à l'énoncé. Cependant, cette bipartition se limite à l'énoncé : on pose comme hypothèse que tous les cas où l'opération dés-orientante de la ponctuation est repérable à un niveau supérieur à celui de l'énoncé renvoient à des configurations de l'indicible.

\section{Niveau énoncé : ponctuation co-orientante /vs/ ponctuation dés-orientante}

La ponctuation remplit essentiellement deux fonctions (Anis, 1988).

4 a) La ponctuation à fonction syntaxique ([alinea] [.] [ ;] [ :] [,]) segmente le continuum scriptural, délimite les syntagmes en les séparant, et enfin les hiérarchise en indiquant la nature de leur relation. C'est ainsi que la [,] indique l'opposition entre la relative déterminative et la relative explicative, oppose l'adverbe d'énoncé à l'adverbe 
d'énonciation (il est mort naturellement / il est mort, naturellement) ; que l'opposition [,] / [;] recompose la structure syntaxique (Pierre a rencontré Jeanne, Claude, Ernest / Pierre a rencontré Jeanne ; Claude, Ernest).

5 b) La ponctuation polyphonique comprend l'ensemble des signes qui indiquent un décrochage énonciatif, qu'il s'agisse des marques du discours cité : [«»], [italique], [tiret de dialogue], des marqueurs expressifs : [capitale], [soulignement], [italique], [tiret] ; des hiérarchiseurs discursifs : [ :] [- -] [( )]; ou des marqueurs de décrochage énonciatif : [?] [! ] [...].

6 La ponctuation syntagmatique opère de manière co-orientante par rapport à l'énoncé : le jeu de comparaison entre énoncés à mots et ordre de mots identiques a pour vertu de montrer que la ponctuation, dans ce cas, figure la construction syntaxique en générant ses modes de reconnaissance. Dans la production de tout autre énoncé, elle agit conjointement aux autres opérateurs syntaxiques.

7 Dans la ponctuation polyphonique, marqueurs expressifs et marqueurs de décrochage énonciatif fonctionnent de manière dés-orientante par rapport à l'énoncé, dans la mesure où ces signes, ou bien excèdent le sens donné par l'énoncé (marqueurs expressifs), ou bien le détournent (signes de modalité). Je ne retiendrai que les signes indicateurs de modalité, dont les énoncés qu'ils affectent, parce qu'ils obligent à ne pas prendre le dit au pied de la lettre, sont comme des passerelles pour d'autres sens. Pour illustrer cette opération, je m'intéresserai ici au [ ?] et au [ !], mais elle vaut également pour les [...] et, globalement, pour les marqueurs expressifs ${ }^{1}$.

Soit les énoncés suivants :

$9 \quad$ (a) Cette décision est inadmissible, tu entends ?

10 (b) Vous partez déjà, monsieur Dupont?

11 Acte de parole indirect, ce type d' interrogation répond à des intentions autres que celles qu'on prête généralement au fait de poser une question. En effet, dans (a) tu entends?, l'interrogation porte sur le dire et non sur le dit, ce qui explique le changement de sens du verbe entendre (il ne s'agit pas d'une interrogation sur le fait de percevoir par l'ouïe ou sur le fait de comprendre ce qui vient d'être dit, mais d'une manière d'interdire à l'interlocuteur toute riposte. Dans (b), le fait de l'interrogation détourne encore une fois la littérarité de l'énoncé, car il serait incohérent de demander une information sur ce qui est de fait constaté : sous couvert de l' énoncé interrogatif, l'énonciateur porte un jugement sur le départ sous l'angle des convenances.

Le [!] fonctionne différemment dans son rapport à l'énoncé qu'il affecte. «Expression du plus haut degré » (Culioli), le [!] est en quelque sorte le substitut idéographique de l'expression du superlatif absolu, ou mieux : se produit un transbordement du sens, qui pourrait être donné par les signes linguistiques, sur le [!], signe idéographique. Il modifie donc sensiblement le sens, pourtant complet, véhiculé par la chaîne alphabétique. Mais s'agit-il d'un simple transfert? A comparer les deux énoncés suivants que l'on pourrait $a$ priori considérer sémantiquement semblables: Prenez la porte! / je vous ordonne de prendre la porte., on perçoit d'emblée que l'effet de sens n'est pas le même. En effet, la dimension pragmatique du [!] en fait à la fois un modificateur et un translateur de sens : l'opération qu'il effectue porte toujours sur le rapport de l'énonciateur à ce qu'il dit, de sorte que l'énoncé exclamatif renvoie au moins autant à l'énonciateur qu'à un référent. Ainsi, (a) Il fait froid! (b) Jean est bel homme!, je constate moins qu'il fait froid que je ne parle de moi dans mon rapport au froid; de la même façon, j'évoque moins la beauté de Jean que ma 
sensibilité à sa beauté. Bref, je parle de moi, je me parle. D'où l'effet de sens différent dans Prenez la porte! / Je vous ordonne de prendre la porte., car dans l'injonction du premier énoncé se manifeste tout l'investissement émotionnel $\mathrm{du}$ 《je » dont est parfaitement dénué le second énoncé.

Ce qui est donc remarquable dans l'énoncé interrogatif ou exclamatif, c'est qu'il renvoie de façon spectaculaire à l'énonciateur. Que la polyphonie instaurée par la ponctuation opère dans le deux -[?]- ou dans l'un -[!]- du sujet (mais il est clair que la polyphonie dans l'un se répercute chez l'interlocuteur), elle produit un effet dés-orientant par rapport au sens référencié. Ce retour de l'énonciation sur elle-même entre en interaction avec le discours in praesentia pour produire un certain dire qui affecte le sujet dans le discours énoncé par le sujet.

Cette analyse a voulu démontrer les mécanismes ponctuationnels de dés-orientation par rapport à l'énoncé référencié ; c'est pourquoi elle s'est fondée sur des énoncés fabriqués, également valables en langue. Tout porte à croire que la ponctuation, en tant que forme de l'indicible, connaît un phénomène de déplacement comparable, mais susceptible de s'étendre à tous les signes, à fonction polyphonique et à fonction syntaxique. Ce qui peut être schématisé ainsi :

\begin{tabular}{|c|c|c|}
\hline $\begin{array}{l}\text { Au niveau } \\
\text { de l'énoncé }\end{array}$ & $\begin{array}{l}\text { - Ponctuation syntagmatique : } \\
\text { orientante par rapport au sens } \\
\text { référencié } \\
\text { - Ponctuation polyphonique: dés- } \\
\text { orientante par rapport au sens } \\
\text { référencié }\end{array}$ & $\begin{array}{l}\text { Sous l'effet de la norme, la ponctuation } \\
\text { polyphonique est perçue elle aussi } \\
\text { comme orientante. }\end{array}$ \\
\hline $\begin{array}{l}\text { Au niveau } \\
\text { textuel }\end{array}$ & $\begin{array}{l}\text { - Ponctuation syntagmatique et } \\
\text { polyphonique: dés-orientante par } \\
\text { rapport au sens référencié }\end{array}$ & $\begin{array}{l}\text { - Sous l'effet de la norme (de l'écrit tout } \\
\text { venant), les deux ponctuations sont } \\
\text { perçues comme dés-orientantes. }\end{array}$ \\
\hline
\end{tabular}

Cependant, pour ce qui concerne l'indicible, il faut nécessairement que la ponctuation en porte l'indice : l'opération de dés-orientation se produit, à mon sens, par la fréquence (surou sous-ponctuation, jugée comme telle par rapport à une moyenne empiriquement perceptible ${ }^{2}$ ) et/ou le site des signes, de sorte que la syntaxe ou le rythme, selon, apparait concurrencer, par la manifestation de leur prégnance, le sens référencié alors empêché de signifier de façon à la fois pleine et autonome.

\section{Au niveau textuel : la ponctuation dés-orientante}

Parce qu'elle fournit des instructions de lecture et limite les effets d'ambiguïté, la fonction première de la ponctuation est la lisibilité. La ponctuation dés-orientante, en déclinant ses modes de dérivation, ne remplit pas cette fonction, ou plutôt, elle implique une lisibilité d'un autre type, lisibilité qui s'instaure paradoxalement dans le trouble du sens représenté, lisibilité de l'indicible.

analysera maintenant la ponctuation dans son effet dés-orientant. On s'attachera singulièrement à la [,] dans L'innommable de Beckett. Il s'agit donc d'un texte littéraire et on ne s'en étonnera pas, car si tout écrit est prédéterminé par les paramètres qui le 
conditionnent (destinateur, destinataire, but, contexte social), la prose poétique a ceci pour elle qu'elle n'est pas astreinte à la "clarté » ou à "l'efficacité » : elle se distingue précisément des écrits ordinaires par le point de cessation du manque à s'écrire (J.-C. Milner, 1978: 38). La ponctuation, chez Beckett, mais pour d'autres écrivains également, constitue notoirement le point de cessation.

18 "C'est de moi maintenant que je dois parler, fût-ce avec leur langage ", dit le locuteur de L'innommable (62). Ce devoir imposé est voué à l'impasse, car comment se (re)connaître si je suis référé à un langage que je n'habite pas, un langage que je peux le cas échéant employer utilitairement mais dont je sais qu'il ne me réfléchit pas? Si je n'ai pas incorporé ce langage que je ne reconnais donc pas comme mien, comment l'identité du moi peut-elle seulement être présupposée ? Le roman de Beckett avance au gré de l'exploration de cette aporie.

19 A mon sens, ce n'est pas tant à travers les mots que se reconnaîtra cette coupure entre le moi et ce langage qui n'est pas mien, mais à travers la ponctuation qui, avec la syntaxe et l'intonation, montrera la distance étrangère. Les mots seront reconnus dans leur renvoi à leur référent, mais ne le seront pas par la bouche et l'oreille du narrateur beckettien qui les profère.

Sur la longueur du roman, on constate une évolution du geste ponctuationnel dont le mouvement même constitue le parcours de cette exploration. Le clavier ponctuationnel, limité à quatre signes -[§] [ ?] [.] [,] $]^{4}$ sera assez rapidement réduit de moitié :

(a) Le [\$] suivi d'un double interligne disparaît dès la fin du préambule. Or, la mise en forme paragraphique, qui rend visuellement compte de l'organisation thématique majeure à partir de laquelle se construit la cohésion, coïncide avec le fait que le narrateur est entouré de comparses. Que cette compagnie soit plus ou moins douteuse aux yeux même du narrateur importe peu, car elle suffit à composer avec l'autre : cette négociation semble ici avoir partie liée avec la structuration paragraphique de l'information, comme si l'autre représenté poussait le sujet écrivant à une relative observance des contraintes communicationnelles (ici, en l'occurrence, ponctuationnelles).

(b) Le [ ?], bien plus résistant, tient la longueur sur les neuf dixièmes du roman ${ }^{5}$. Sa disparition coïncide avec l'auto-dépouillement des autres moi de substitution (MahoodWorm), définitivement supplantés par un tiers collectif indéterminé et persécuteur (ils; eux) dont la présence croissante pousse le je dans ses derniers retranchements.

(c) La fin du roman atteint un point de non retour de la pleine conscience du moi morcelé, dont l'éparpillement dit le moi exilé de sa propre voix. Mais les mots ne suffisent pas à donner toute la mesure de cette débâcle, ils ne font qu'en donner la représentation, ils ne l'atteignent pas. La ponctuation dés-orientante est produite par un faire ponctuationnel qui, en réalisant performativement la coupure d'avec la voix, fait affleurer l'indicible de la réalisation, du faire-advenir même de cette coupure. L'indicible de, paradoxalement, son incorporation, la coupure de la voix faite je. Comment?

On a vu que l'exclusion successive des signes figure le mouvement centripète du je au centre de son absence de corps et de voix : dans son absence radicale à lui-même. Le [.] et la [,] sont les seuls signes restants. Cependant, l'occurrence du point s'espace considérablement, dans un rapport inversement proportionnel à la virgule dont la fréquence particulièrement élevée s'explique par la brièveté des syntagmes propositionnels juxtaposés. Les unités discursives ${ }^{6}$ présentent toutes un faible volume et redupliquent à peu d'exceptions près la même structure syntaxique, de sorte qu'à 
l'intérieur de ces périodes distendues à l'extrême (raréfaction de l'intonème conclusif) se reproduit systématiquement le même patron intonatoire ${ }^{7}$.

L'endroit, je le ferai quand même, je le ferai dans ma tête, je le tirerai de ma mémoire,

je le tirerai vers moi, $\overline{j e ~ m e ~ f e r a i ~ u n e ~ t e ̂ t e, ~} \widehat{j e ~ m e ~ f e r a i ~ u n e ~ m e ́ m o i r e, ~} \overline{j e ~ n ' a i ~ q u ' a ̀ ~ e ́ c o u t e r ~}$

\section{$[\ldots]($ p. 207)}

De loin en loin le niveau grave (intonème conclusif du [.]), et de façon très rapprochée le niveau infra-aigu (intonème continuatif de la [,]). Ce patron intonatoire est en soi tout-àfait conforme ${ }^{8}$, mais sa réitération, invariable, consécutive, étendue sur plusieurs pages, l'est beaucoup moins dans la mesure où, sur la longueur d'une séquence textuelle, elle ne correspond à aucun schéma acoustique. Le jeu accentuel, lui aussi inhabituel, va dans le sens de cette perte de repère. On avait remarqué que les unités discursives correspondent à de rares exceptions près à des propositions juxtaposées. Cela signifie qu'en l'absence d'articulateur, il y a de part et d'autre de la [,], ponctuation faible, un changement de hauteur important, qui appelle systématiquement un accent d'attaque à l'ouverture de la nouvelle proposition. On a donc une structure intono-accentuelle avec début de syntagme caractérisé par [brève accentuée + niveau medium -dû à l'accent d'attaque-] et fin du syntagme caractérisé par [longue accentuée + niveau infra-aigu]. En outre, on a affaire exclusivement à des montées, cette structure génère une tension prosodique dont elle ne se départit pas.

Enfin, l'absence de complémentarité [,] / articulateur crée un effet référentiel paradoxal. Si d'une part, les unités discursives s'avèrent référentiellement stabilisées, il semble d'autre part que par manque de hiérarchisation et d'extension syntagmatique, celles-ci ne s'articulent ni ne se fixent au niveau du traitement de l'information. En somme, il y aurait explicitation de l'effritement du moi, mais dans l'effritement textuel même. Du sens traverse le sujet qui ne sait le retenir ni l'organiser.

On voit comment la ponctuation, par la prégnance même de ce qu'elle fait, entre dans sa fonction de dés-orientation par rapport au sens référencié :

- dans sa relation à la syntaxe, la complète mise à plat des propositions, le pur déroulement des contenus représentés, empêchent le sens de s'implanter ou plutôt de s'organiser par apports successifs. C'est en cela que la ponctuation virgulaire, de surcroît dépourvue d'articulateurs, produit une résistance ;

- dans sa relation à la prosodie, elle crée une tension extrême que les mots ne prennent guère en charge. Cette tension provient de la réalisation intonatoire (car il n'y a d'énoncé qu'intoné), vocale, qui ne se soutient d'aucune expérience antérieure, ni d'aucun souvenir acoustique.

Or, il y a là un effet paradoxal car, si d'un côté le sujet narrateur semble prendre toute la mesure de l'éparpillement de son moi, si donc le langage semble ici couvrir le champ de l'intenté, de l'autre, le jeu intono-accentuel déployé par le concours de la ponctuation et de la syntaxe, parce qu'il est remarquable, prend en charge une part que l'on peut interpréter comme ce qui dans le langage fait résistance : l'intonation joue en effet son rôle de donateur de l'énoncé, car, pour reprendre Culioli, il n'y a d'énoncé qu'intoné, mais 
elle produit en même temps un effet déviant qui semble la désolidariser d'avec l'énoncé. Par sa valeur de différenciation, elle produit un surplus, un second pôle, alors qu'elle est à la fois la source et la condition de la production langagière. Evénement pour le moins étrange, où le point de génération même de la parole se disjoint, par son effet de désorientation, de la parole qu'elle engendre pourtant.

\section{Déterritorialisation vocale}

\section{Comment interpréter cet effet paradoxal?}

On considère avec A.C. Berthoud que «le discours est constitué de deux grands types d'actes, soit des actes qui régulent le dire et des actes qui régulent les objets du discours » (Berthoud, 1996 : 141). Cependant, si dans le discours tout-venant les actes qui régulent le dire et ceux qui régulent les objets du discours se conjoignent pour produire le sens, ce n'est pas le cas lorsque la ponctuation est dés-orientante par rapport au sens référencié. Ainsi dans L'innommable, où cette double négociation se trouve comme séparée, ou en tout cas décalée, du fait que l'intonation produit de la non-mémoire acoustique. Pour que les objets du discours soient pleinement identifiables, le référent à lui seul n'est pas suffisant : il faut que l'intonation lui soit congruente, et on a vu que si elle l'était dans les limites propositionnelles, elle ne l'est plus à l'épreuve du temps, c'est-à-dire sur l'étendue des ensembles de propositions.

1 Chez Beckett, le geste ponctuationnel pourrait s'identifier à de l'éprouvé d'une expérience sur lequel le langage, parce qu'il n'a pas prise, n'a de valeur fiduciaire que résiduelle. La construction du sens débouche sur une certaine représentation du sujet -du je qui prend la parole- mais non sur la (dé)construction du sujet qui s'effectue par le biais de la ponctuation. Les mots disent, la ponctuation parle, ce à quoi les mots n'accèdent pas : elle devient un système de dispersion, l'opération en acte du morcellement du moi dont le sens référencié ne constitue qu'un épiphénomène.

Ce à quoi les mots n'accèdent pas, c'est au procès même de la déterritorialisation de la voix, qui tombe sous l'irreprésentable. La voix garde en elle la trace de l'origine (Vasse : 216), premier lien avec le monde, première (re)connaissance de moi et de l'autre. Si la voix du sujet ne parle plus à son oreille, si son oreille n'identifie plus sa voix, l'acte de non recevoir du sujet en tant que tel est signé : la première peau, «la peau auditivophonique » (Anzieu), se défait. Sous l'effet de cette décomposition, tous les repères sont brouillés - identité/altérité, lieu/corps, chronologie de l'avant et de l'après - pour se résorber en un point central repéré sur le tranchant de la voix et de l'écoute, celui de l'origine et de la fin conçus chez Beckett comme un seul et même butoir.

Le geste ponctuationnel dans L'innommable configure une parole d'emblée non identifiable du fait qu'elle est à sa source séparée de toute mémoire intonative et auditive. L'entreprise de la parole consiste ici à historiciser le silence, mais reste toujours ce surplus, cet en-trop, le sujet lui-même, fût-il morcelé. C'est en ce sens que l'on peut dire que la forme de l'indicible est repérable, moins dans la bipartition entre le sens référencié qui prend en charge l'histoire du sujet et la ponctuation comme hiéroglyphe d'une histoire qui cherche à se faire entendre avant les mots, que dans la tentative de faire involuer l'histoire du sujet jusqu'à sa résorption dans celle du silence. Mais en dernière instance, un silence saturé de langage. 


\section{BIBLIOGRAPHIE}

ANIS J., CHISS J.-L., PUECH Ch. (1988) L'écriture. Théories et descriptions, Bruxelles, De Boeck Université.

BECKETT S. (1953) L'innommable, Paris, Minuit.

BERRENDONNER A. (1993) La phrase et les articulations du discours, Le Français dans le monde, Recherches et applications, février-mars.

BERTHOUD A.-C. (1996) Paroles à propos. Approche énonciative et interactive du topic, Paris, Ophrys, Coll. L'homme dans la langue.

MILNER J.-C. (1978) L'amour de la langue, Paris, Seuil.

\section{NOTES}

1. Pour les [...], voir V. Dahlet (1995).

2. On exclut donc d'emblée le discours intérieur dont l'absence de ponctuation répond à une stratégie délibérée.

3. Ces termes, par référence à l'ouvrage bien portant de A. Doppagne (1984), La bonne ponctuation. Clarté, précision, efficacité de vos phrases. Paris-Gembloux : Duculot.

4. S'ajoutent en réalité deux autres signes : le [!], dont les trois occurrences, très rapprochées, modalisent le discours d'un tiers collectif doté du seul trait sémantique /humain/. Discours qui atteint du seul fait de la ponctuation le comble de l'obscénité «de leur vie d'homme» qui se résigne sereinement à l'absence de justification de l'humain (Le voilà des nôtres!) (p. 48); et l'unique [()] qui contient comme un rappel : je pense toujours (p. 95).

5. Sauf erreur, le dernier [ ?] apparait p. 184, sachant que le roman compte 213 pages.

6. Pour une analyse des unités discursives et des périodes /vs/ le concept abstrait de phrase, cf. Berrendonner A., 1993, «La phrase et les articulations du discours». Le Français dans le monde, Recherches et applications, Des pratiques de l'écrit, février-mars.

7. Les lignes obliques renvoient au schéma intonatoire, l'italique, à l'accent d'attaque et les caractères gras à l'accent de fin de groupe.

8. A défaut d'enregistrement, la description du patron intonatoire est évidemment très approximative, mais il reste par-delà les variations possibles un invariant de base. Pour M. Rossi et alii, «Le continuum prosodique est interprété [...] par des mécanismes de perception qui opèrent une sorte de filtrage pour retrouver [...] les traits liés aux instructions linguistiques» (1981: 89). 


\section{RÉSUMÉS}

Marque d'un manque à référer, l'indicible procède de la même manière que la métaphore. Or certains modes d'actions de la ponctuation sur le référé, que l'on analysera comme un processus de non congruence, autorisent à les identifier comme une forme de l'indicible. Cette contribution cherche à en analyser les modalités dans l'innomable de S. Beckett.

The unsayable, as a mark of impossibility to refer, proceeds in the same way as a metaphor. Certain effects of ponctuation on the referent, which are analysed as a process of incongruence, allow us to identify them as a form of unutterability. This paper attempts to analyse these types of modality in Beckett's L'innommable.

\section{AUTEUR}

\section{VÉRONIQUE DAHLET}

Université de São-Paulo (USP) 